\title{
Several Types of Somatic Mutations Induce Autonomous Production of Aldosterone in Primary Aldosteronism
}

\author{
Tetsuo Nishikawa*, Takumi Kitamoto, Yoko Matsuzawa, Jun Saito and Masao Omura
}

Endocrinology and Diabetes Center, Yokohama Rosai Hospital, Yokohama, Japan

Received: May 30, 2014; Accepted: May 31, 2014; Published: June 02, 2014

*Corresponding author: Tetsuo Nishikawa, Endocrinology and Diabetes Center, Yokohama Rosai Hospital, 3211 Kozukue-cho, Kohoku-ku, Yokohama City, Kanagawa 222-0036, Japan, Tel: 81-454748111; Fax: 81-454748323; E-mail: tetsuon@yokohamah.rofuku.go.jp

It is well known that primary aldosteronism (PA) is the most common form of secondary hypertension, and also that aldosterone-producing adenoma (APA) and bilateral hyperaldosteronism (idiopathic hyperaldosteronism: IHA) are the most common forms of PA. Now, genetic analysis for solving pathogenesis of PA is progressing. Somatic gene mutations of the selectivity filter of the potassium $\mathrm{K}(+)$ channel KCNJ5 were recently detected in APA in patients with sporadic PA [1]. These mutations were reported to produce increased sodium $(\mathrm{Na}+)$ conductance and cell depolarization resulting in autonomous aldosterone production in the APA [1]. It was already reported that somatic G151R or L168R mutations were found in $40 \%$ of APA associated with marked hyperplasia, but not in specimens with merely unilateral hyperplasia [2]. Moreover, it had recently been reported that the TASK- 2 channel lower expression represents universal characteristics of APA, associated with a higher expression of hsa-miR-23 and hsa-miR-34. The blunted TASK-2 activity induced an increase in the production of aldosterone in vitro and the expression of steroidogenic acute regulatory protein and CYP11B2.Thus, the lower expression of TASK-2 channel in APA cells can explain autonomous production of aldosterone in PA [3]. Gomez-Sanchez [4] had recently summarized that somatic mutations of the selectivity filter of the Kir3.4 channel in APA results in loss of selectivity for $\mathrm{K}(+)$ and entry of sodium, resulting in membrane depolarization, calcium mobilization, increased CYP11B2 expression, and hyperaldosteronism. Thus, he emphasized that APA is caused by channelopathies inducing an increase in aldosterone synthesis [4]. Germ cell mutations of KCNJ5 cause familial hyperaldosteronism type 3, which is associated with adrenal zona glomerulosa hyperplasia, rather than adenoma [5,6]. Less commonly, somatic mutations of the sodium-potassium ATPase, calcium ATPase, or the calcium channel (calcium channel voltage-dependent L type alpha 1D) have been found in some APAs. It was also reported that somatic mutations in ATP1A1, ATP2B3, and KCNJ5 were present in $6.3 \%, 0.9 \%$, and $39.3 \%$ of APAs, respectively, and included 2 novel mutations $(\mathrm{Na}(+) / \mathrm{K}(+)$-ATPase p.Gly99Arg and GIRK4 p.Trp126Arg) [7]. CYP11B2 gene expression was higher in APAs harboring ATP1A1 and ATP2B3 mutations compared with those without these or KCNJ5 mutations [7].
We detected a low CYP17/HSD3B2 ratio in APA tissue, indicating that the cortisol pathway is suppressed in APA and also observed a high CYP11B2/CYP11B1 ratio in APA tissue, suggesting that the aldosterone pathway is accelerated in APA [8]. Furthermore, we reported that there were no KCNJ5 gene mutations in the normal tissue adhering to KCNJ5 gene mutationharboring APA, suggesting that somatic mutations of the KCNJ5 gene only occur in APA tissue [9]. On the other hand, we had clearly demonstrated that APA cells produce markedly less cortisol than normal adrenal cells, and it is suggested that KCNJ5 gene mutations dominantly induce the aldosterone production pathway as well as a concomitant decline in cortisol production, resulting in highly autonomous aldosterone production [9]. It is possible to consider that accelerated aldosterone production in APA is caused mainly by channelopathies, as reported by Gomez-Sanchez CE [4]. Those channelopathies may increase calcium entry inside adrenal cells, activating Ca-dependent intracellular signaling systems related to regulating aldosterone synthesis. Then we have questions how to treat the patients with PA possessing those channelopathies, to do surgery or not? We should perform adrenal vein sampling (AVS) before surgical treatment because of precise detection of unilateral adrenal lesions, while AVS cannot always be done for all patients with PA because of inadequate numbers of AVS-radiologists. Is it possible to use calcium channel blockade for medical treatment? Moreover, selective CYP11B2 inhibitors have recently been reported, which could be promising drug candidates for the treatment of aldosterone related diseases [10-12]. Then, new treatment, such as local injection of specific CYP11B2 inhibitors should also be considered for adrenal lesions that cause hyperaldosteronism. Moreover, we should precisely evaluate the long-term efficacy of MR antagonists for hyperaldosteronism[13].

Finally, we would like to recommend minimal invasive surgery after precisely detecting adrenal lesions by AVS performed by well-trained radiologist until well-tolerated medical treatment, inducing complete remission, will be developed.

\section{References}

1. Choi M, Scholl UI, Yue P, Bjorklund P, Zhao B et al. (2011) K+ channel 
mutations in adrenal aldosterone-producing adenomas and hereditary hypertension. Science 331(6018): 768-772

2. Akerström T, Crona J, Delgado Verdugo A, Starker LF, Cupisti K,et al. (2012) Comprehensive re-sequencing of adrenal aldosterone producing lesions reveal three somatic mutations near the KCNJ5 potassium channel selectivity filter. PLoS One: DOI 10.1371.

3. Lenzini L, Caroccia B, Campos AG, Fassina A, Belloni AS, et al. (2014) Lower Expression of the TWIK-Related Acid-Sensitive $\mathrm{K}(+)$ Channel 2 (TASK-2) Gene Is a Hallmark of Aldosterone-Producing Adenoma Causing Human Primary Aldosteronism. J Clin Endocrinol Metab 99(4): 674-682.

4. Gomez-Sanchez CE, Oki K (2014) Minireview: potassium channels and aldosterone dysregulation: is primary aldosteronism a potassium channelopathy? Endocrinology 155(1): 47-55.

5. Quack I, Vonend O, Rump LC (2010) Familial hyperaldosteronism I-III. Horm Metab Res 42(6): 424-428.

6. Monticone S, Hattangady NG, Penton D, Isales CM, Edwards MA et al. (2013) a Novel Y152C KCNJ5 mutation responsible for familial hyperaldosteronism type III. J Clin Endocrinol Metab 98(11): 1861-1865.

7. Williams TA, Monticone S, Schack VR, Stindl J, Burrello J, et al. (2014) Somatic ATP1A1, ATP2B3, and KCNJ5 mutations in aldosteroneproducing adenomas. Hypertension 63(1): 188-195.
8. Sakuma I, Suematsu S, Matsuzawa Y, Saito J, Omura M, et al. (2013) Characterization of steroidogenic enzyme expression in aldosteroneproducing adenoma: a comparison with various human adrenal tumors. Endocr J 60: 329-336.

9. Matsuzaswa Y, Kitamoto T, Suematsu S, Saito J, Omura M, et al. (2014) Steroidogenic activity of aldosterone-producing adenoma with and without KCNJ5 gene mutations, comparing with that in each adherent normal tissue. J Endocrinol Diab 1(1): 1-6.

10. Schumacher CD, Steele RE, Brunner HR (2013) Aldosterone synthase inhibition for the treatment of hypertension and the derived mechanistic requirements for a new therapeutic strategy. J Hypertens 31(10): 2085 2093.

11. Andersen K (2013) Aldosterone synthase inhibition in hypertension. Curr Hypertens Rep. 15(5): 484-488.

12. Hu Q, Yin L, Hartmann RW (2014) Aldosterone synthase inhibitors as promising treatments for mineralocorticoid dependent cardiovascular and renal diseases. J Med Chem. Date of Electronic Publication: 2014 Jan. 24.

13. Nishikawa T, Omura M, Saito J, Matsuzawa Y (2012) Primary aldosteronism: comparison between guidelines of the Japanese and the US Endocrine Society. Expert Review of Endocrinology \& Metabolism 7(6): 637-645. 